\title{
NUMERICAL SOLUTIONS OF STEADY FREE CONVECTIVE FLOW IN A RECTANGULAR REGION WITH DISCRETE WALL HEAT AND CONCENTRATION SOURCES
}

\author{
Vusala Ambethkar, Lakshmi Rani Basumatary \\ Department of Mathematics, Faculty of Mathematical Sciences \\ University of Delhi, Delhi-110007, India \\ vambethkar@maths.du.ac.in,vambethkar@gmail.com \\ lrbasumatary@gmail.com \\ Received: 16 October 2019; Accepted: 21 January 2020
}

\begin{abstract}
In this paper, numerical solutions are obtained for steady free convective flow in a rectangular region with discrete wall heat and concentration sources by using the finite volume method. The governing equations consist of the continuity, momentum, energy and mass transfer. These equations conjointly with suitable boundary conditions are solved numerically by using this method. The novel concept in this work is to generalize the SIMPLE algorithm suitably and thereby compute the numerical solutions of the flow variables such as the temperature $(\theta)$ and the concentration $(C)$ in addition to the components of velocity and the pressure. All non-dimensional parameters are chosen suitably in accordance with the physical significance of the problem under investigation. With the help of these numerical solutions, we have depicted the profiles of the velocity, pressure, temperature and concentration along the horizontal and vertical directions of the geometric centre of the region. The validity of the numerical solutions are ensured by comparing the present solutions with the benchmark solutions. Code validation has been given for the present problem.
\end{abstract}

MSC 2010: 76D05, 76M12, 80A20

Keywords: control volume, discretized, temperatures, concentration, free convective flow, heat and concentration sources, horizontal and vertical directions

\section{Introduction}

Free convection in a rectangular region due to heat sources placed on its walls is attaining immense significance in the present era's research. The reason for this significance is due to numerous industrial applications of free convection in a rectangular region such as in furnaces, cooling towers, electronic cooling systems et cetera.

A normal time advancing method for evaluation of flow fields of a regular fluid flow problem was deliberated by Patankar and Spalding [1]. Ghia et al. [2] have recommended benchmark solutions for a $2 \mathrm{D}$ incompressible flow in a square cavity by using the multigrid method. The benchmark solution for free convective flow of 
air in a square cavity was studied by Davis [3]. Bejan [4] investigated heat and mass transfer of a free convective flow under buoyancy effects in a rectangular enclosure. Trevisan and Bejan [5] have investigated the heat and mass transfer effects due to free convective flow in a vertical enclosure. Temperature and concentration effects of a free convective flow in a rectangular enclosure was investigated by Phanikumar [6].

Chamkha and Naser $[7,8]$ have studied numerically the unsteady and hydromagnetic double-diffusive convective flow in a rectangular enclosure through a porous medium in the presence of temperature and concentration gradients. Nakhi and Chamkha [9-11] have presented a steady and conjugate incompressible free convective flow in an inclined partitioned enclosure and thin fin with arbitrary lengths. Combined buoyancy effects of thermal and mass diffusion of a mixed convective flow in a rectangular enclosure was studied by Teamah and Maghlany [12]. Sathyamoorthy and Chamkha [13] have investigated the free convective flow in a square cavity due to the magnetic field effect. Kuznetsov and Sheremed [14] have reported numerical simulations of double diffusive free convective flow in an enclosure. Ozotop et al. [15] have investigated numerically the free convective flow with heated vertical wall temperature in a wavy enclosure. The free convective flow in a rectangular cavity with thermally active discrete side wall was investigated numerically by Nikbakti and Rahimi [16]. Qin et al. [17] have reported high accurate numerical solutions in a rectangular enclosure with heat and concentration sources. The problem of steady laminar mixed convective flow in both square and triangular cavity which is filled with water was studied by Ismael et al. [18]. Double-diffusive free convective flow in an open square cavity with partial heating effects was performed numerically by Arbin et al. [19].

A literature survey revealed that there is a significant need for accurate the numerical solutions of the flow variables associated with free convection due to heat and concentration sources placed on the walls of a rectangular region. However, there is a complexity involved in this problem such as the governing equations are a system of coupled quasi-linear partial differential equations conjoined with boundary conditions. Therefore, in the present work, we have attempted to obtain the accurate numerical solutions of the flow variables by using the upwind finite volume scheme.

The novelty in this current investigation is apparent from the generalization of the SIMPLE algorithm so as to compute the numerical solutions of the new flow variables such as the temperature $(\theta)$ and the concentration $(C)$. The original SIMPLE algorithm solves the flow variables such as the components of velocity and pressure but, due to inserting discrete heat and concentration sources on the wall of the rectangle, there is a need to compute the numerical solutions of the temperature $(\theta)$ and the concentration $(C)$. Consequently, there is a need to generalized the discretization equations for the temperature $(\theta)$ and the concentration $(C)$ variables and finally we compute their numerical solutions with the help of this generalized SIMPLE algorithm.

The main objective of this study is to discretize the governing quasi-linear partial differential equations by using the upwind finite volume scheme. We compute the 
numerical solutions of the flow variables by using the SIMPLE algorithm. We compare the numerical solutions obtained in this study with the benchmark solutions available in the literature. Hence, we conclude that our numerical solutions are accurate and realistic.

\section{Formulation of the problem}

\subsection{Description}

The description of free convective flow in a rectangular region with discrete heat and concentration sources on its walls is given in Figure 1. $A B C D$ is a rectangular region of length $L$ and height $H$ in which free convective flow is considered [20]. We have assumed that the top wall is moving towards right direction while other three walls are stationary. Therefore, no-slip boundary conditions for velocity are defined on these walls. A discrete heat and a concentration source is placed on the bottom wall of the rectangular region [20]. We have assumed the bottom wall is hot and top wall is cold. Therefore, the temperature and concentration on the bottom and the top wall of the region $\theta_{h}, \theta_{c}, C_{h}$ and $C_{c}$ [20]. At all the corner points of the region, $(u, v)$ and $P$ are assumed to be zero. Excluding the corner points, the boundary conditions for velocity, temperature and concentration on all boundary walls of region $A B C D$ are defined as shown below.

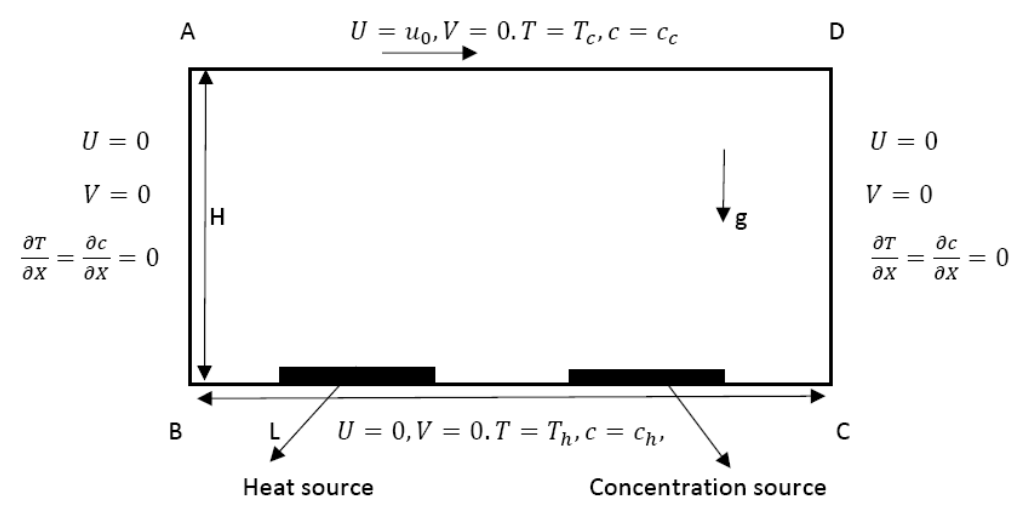

Fig. 1. Diagram of physical problem

\subsection{Governing equations}

The governing equations of a free convective flow in a rectangular region with heat and concentration effects are described by the continuity equation, momentum equations in $X$ and $Y$ directions, the energy and mass transfer equations in dimensional form are given as follows [20]:

$$
\text { continuity equation: } \quad \frac{\partial U}{\partial X}+\frac{\partial V}{\partial Y}=0,
$$


$X$-momentum equation:

$$
U \frac{\partial U}{\partial X}+V \frac{\partial U}{\partial Y}=-\frac{1}{\rho} \frac{\partial p}{\partial X}+v\left(\frac{\partial^{2} U}{\partial X^{2}}+\frac{\partial^{2} U}{\partial Y^{2}}\right)
$$

$Y$-momentum equation:

$$
\begin{aligned}
& U \frac{\partial V}{\partial X}+V \frac{\partial V}{\partial Y}=-\frac{1}{\rho} \frac{\partial p}{\partial Y}+v\left(\frac{\partial^{2} V}{\partial X^{2}}+\frac{\partial^{2} V}{\partial Y^{2}}\right) \\
& +g\left[\beta_{T}\left(T-T_{c}\right)+\beta_{c}\left(c-c_{c}\right)\right],
\end{aligned}
$$

energy equation:

$$
U \frac{\partial T}{\partial X}+V \frac{\partial T}{\partial Y}=\alpha\left(\frac{\partial^{2} T}{\partial X^{2}}+\frac{\partial^{2} T}{\partial Y^{2}}\right)
$$

mass transfer equation:

$$
U \frac{\partial c}{\partial X}+V \frac{\partial c}{\partial Y}=D\left(\frac{\partial^{2} c}{\partial X^{2}}+\frac{\partial^{2} c}{\partial Y^{2}}\right) \text {. }
$$

where $U, V, P, \rho, T, g, \beta_{T}, \beta_{c}, \alpha, c$ and $D$ are the components of velocity along the $X$ and $Y$ axis, pressure, density, temperature, gravitational acceleration, thermal expansion coefficient of the fluid, solutal expansion coefficient of the fluid, thermal diffusivity, concentration and mass diffusivity respectively.

Introducing the dimensionless variables as:

$$
\begin{aligned}
& (x, y)=\frac{(X, Y)}{L},(u, v)=\frac{(U, V)}{u_{0}}, P=\frac{p}{\rho u_{0}^{2}}, \theta=\frac{T-T_{c}}{T_{h}-T_{c}}, C=\frac{c-c_{c}}{c_{h}-c_{c}}, \operatorname{Pr}=\frac{v}{\alpha}, S c=\frac{v}{D}, \\
& R e=\frac{u_{0} L}{v}, R i=\frac{G r}{R e^{2}}, G r=\frac{g \beta_{T}\left(T_{h}-T_{c}\right)}{\gamma^{2}} L^{3}, N=\frac{\beta_{c}\left(c_{h}-c_{c}\right)}{\beta_{T}\left(T_{h}-T_{c}\right)} .
\end{aligned}
$$

The dimensional boundary conditions for the governing equations (1)-(5) are:

$$
\left.\begin{array}{l}
\text { on } \mathrm{AB}: \quad \text { at } X=0, U=V=0, \frac{\partial T}{\partial X}=\frac{\partial c}{\partial X}=0, \\
\text { on } \mathrm{DC}: \quad \text { at } X=H, U=V=0, \frac{\partial T}{\partial X}=\frac{\partial c}{\partial X}=0, \\
\text { on } \mathrm{BC}: \quad \text { at } Y=0, U=V=0, T=T_{h}, c=c_{h}, \\
\text { on } \mathrm{AD}: \quad \text { at } Y=L, U=u_{0}, V=0, T=T_{c}, c=c_{c} .
\end{array}\right\}
$$

After using the dimensionless variables, the dimensionless form of the governing equations (1)-(5) reduce to

$$
\begin{aligned}
& \text { continuity equation: } \quad \frac{\partial u}{\partial x}+\frac{\partial v}{\partial y}=0 \\
& x \text {-momentum equation: } \quad u \frac{\partial u}{\partial x}+v \frac{\partial u}{\partial y}=-\frac{\partial P}{\partial x}+\frac{1}{R e}\left(\frac{\partial^{2} u}{\partial x^{2}}+\frac{\partial^{2} u}{\partial y^{2}}\right), \\
& y \text {-momentum equation: } \quad u \frac{\partial v}{\partial x}+v \frac{\partial v}{\partial y}=-\frac{\partial P}{\partial y}+\frac{1}{R e}\left(\frac{\partial^{2} v}{\partial x^{2}}+\frac{\partial^{2} v}{\partial y^{2}}\right)+R i(\theta+N C) \text {, } \\
& \text { energy equation: } \quad u \frac{\partial \theta}{\partial x}+v \frac{\partial \theta}{\partial y}=\frac{1}{\operatorname{RePr}}\left(\frac{\partial^{2} \theta}{\partial x^{2}}+\frac{\partial^{2} \theta}{\partial y^{2}}\right) \text {, } \\
& \text { mass transfer equation: } \quad u \frac{\partial C}{\partial x}+v \frac{\partial C}{\partial y}=\frac{1}{R e S c}\left(\frac{\partial^{2} C}{\partial x^{2}}+\frac{\partial^{2} C}{\partial y^{2}}\right) \text {, }
\end{aligned}
$$

and the boundary conditions in dimensionless form are 


$$
\left.\begin{array}{l}
\text { on } \mathrm{AB}: \quad \text { at } x=0, u=v=0, \frac{\partial \theta}{\partial x}=\frac{\partial C}{\partial x}=0, \\
\text { on } \mathrm{DC}: \quad \text { at } x=2, u=v=0, \frac{\partial \theta}{\partial x}=\frac{\partial C}{\partial x}=0, \\
\text { on } \mathrm{BC}: \quad \text { at } y=0, u=v=0, \theta=1, C=1, \\
\text { on } \mathrm{AD}: \quad \text { at } y=1, u=1, v=0, \theta=0, C=0 .
\end{array}\right\}
$$

where the dimensionless variables $u, v, P, R e, R i, N, \theta, C, P r$ and $S c$ are velocity components along the $x$ and $y$-axis, pressure, the Reynolds number, the Richardson number, the buoyancy ratio, temperature, concentration, the Prandtl number and the Schmidt number respectively.

\section{Numerical method}

\subsection{Discretization}

We discretize the governing equations (7)-(11) by using the finite volume method under a staggered grid arrangement as given in [21] which is described in the following Figure 2.

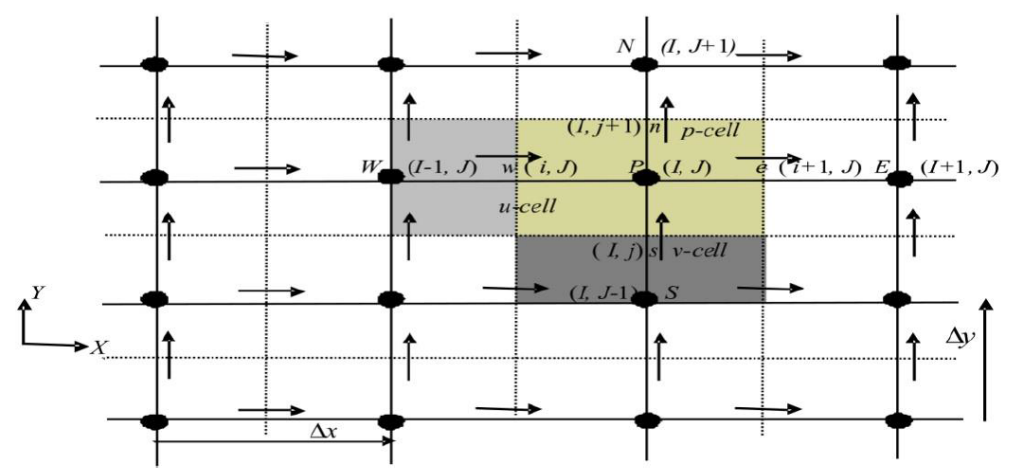

Fig. 2. Staggered grid arrangement

Continuity equation: The discretized continuity equation at location $(I, J)$ of the scalar control volume is given by [21]

$$
F_{e}-F_{w}+F_{n}-F_{s}=0
$$

$x$-momentum: The discretized $x$-momentum equation at location $(i, J)$ of the $u$-control volume is given by [21]

$$
a_{i, J} u_{i, J}=\sum a_{n b} u_{n b}+\left(P_{I-1, J}-P_{I, J}\right) A_{i, J}
$$

where $A_{i, J}$ is the cell face area of $u$-control volume. $E, W, N, S$ neighbors involved in the summation $\sum a_{n b} u_{n b}$ are $(i+1, J),(i-1, J),(i, J+1)$ and $(i, J-1)$. The coefficients of the upwind differencing scheme [21] are

$$
a_{i, J}=a_{i-1, J}+a_{i+1, J}+a_{i, J-1}+a_{i, J+1}+\Delta F
$$


where

$$
\left.\begin{array}{l}
a_{i+1, J}=D_{I, J}+\max \left(-F_{I, J}, 0\right), \quad a_{i-1, J}=D_{I-1, J}+\max \left(F_{I-1, J}, 0\right), \\
a_{i, J+1}=D_{i, j+1}+\max \left(-F_{i, j+1}, 0\right), \quad a_{i, J-1}=D_{i, j}+\max \left(F_{i, j}, 0\right), \\
\Delta F=\left(F_{e}-F_{w}\right)+\left(F_{n}-F_{S}\right)=\left(F_{I, J}-F_{I-1, J}\right)+\left(F_{i, j+1}-F_{i, j}\right)
\end{array}\right\}
$$

For each of the faces $e, w, n$ and $s$, we have

$$
\left.\begin{array}{l}
F_{e}=F_{I, J}=\frac{F_{i+1, J}+F_{i, J}}{2}=\frac{u_{i+1, J} A_{i+1, J}+u_{i, J} A_{i, J}}{2} \\
F_{w}=F_{I-1, J}=\frac{F_{i, J}+F_{i-1, J}}{2}=\frac{u_{i, J} A_{i, J}+u_{i-1, J} A_{i-1, J}}{2} \\
F_{n}=F_{i, j+1}=\frac{F_{I, j+1}+F_{I-1, j+1}}{2}=\frac{v_{I, j+1} A_{I, j+1}+v_{I-1, j+1} A_{I-1, j+1}}{2} \\
F_{S}=F_{i, j}=\frac{F_{I, j}+F_{I-1, j}}{2}=\frac{v_{I, j} A_{I, j}+v_{I-1, j} A_{I-1, j}}{2} \\
D_{e}=D_{I, J}=\frac{A_{I, J}}{\operatorname{Re} \Delta x}=\frac{1}{\operatorname{Re} \Delta x}\left(\frac{A_{i+1, J}+A_{i, J}}{2}\right), D_{w}=D_{I-1, J}=\frac{A_{I-1, J}}{\operatorname{Re} \Delta x}=\frac{1}{\operatorname{Re} \Delta x}\left(\frac{A_{i, J}+A_{i-1, J}}{2}\right) \\
D_{n}=D_{i, j+1}=\frac{A_{i, j+1}}{\operatorname{Re} \Delta y}=\frac{1}{\operatorname{Re} \Delta y}\left(\frac{A_{I, j+1}+A_{I-1, j+1}}{2}\right), D_{s}=D_{i, j}=\frac{A_{i, j}}{\operatorname{Re\Delta y}}=\frac{1}{\operatorname{Re\Delta y}}\left(\frac{A_{I, j}+A_{I-1, j}}{2}\right)
\end{array}\right\}
$$

$y$-momentum: The discretized $y$-momentum equation at $(I, j)$ is given by [21]

$$
a_{I, j} v_{I, j}=\sum a_{n b} v_{n b}+\left(P_{I, J-1}-P_{I, J}\right) A_{I, j}+b_{I, j}
$$

where $A_{I, j}$ is the cell face area of $v$-control volume. The neighbours $E, W, N$ and $S$ involved in the summation $\sum a_{n b} v_{n b}$ are $(I+1, j),(I-1, j),(I, j+1)$ and $(I, j-1)$.

Now the coefficients of the upwind differencing scheme are given [21] as follows:

$$
a_{I, j}=a_{I-1, j}+a_{I+1, j}+a_{I, j-1}+a_{I, j+1}+\Delta F-S_{I, j}
$$

and

$$
S_{I, j} v_{I, j}+b_{I, j}=\bar{S} \Delta v
$$

where

$$
\left.\begin{array}{l}
a_{I+1, j}=D_{i+1, j}+\max \left(-F_{i+1, j}, 0\right), a_{I-1, j}=D_{i, j}+\max \left(F_{i, j}, 0\right), \\
a_{I, j+1}=D_{I, J}+\max \left(-F_{I, J}, 0\right), a_{I, j-1}=D_{I, J-1}+\max \left(F_{I, J-1}, 0\right), \\
\Delta F=\left(F_{e}-F_{w}\right)+\left(F_{n}-F_{S}\right)=\left(F_{i+1, j}-F_{i, j}\right)+\left(F_{I, J}-F_{I, J-1}\right)
\end{array}\right\}
$$

For $v$-control volume, the values of $F$ and $D$ for each of the faces $e, w, n$ and $s$ are defined in a similar manner as in the previous case.

The pressure correction equation is given by [21]

$$
a_{I, J} P_{I, J}^{\prime}=a_{I+1, J} P_{I+1, J}^{\prime}+a_{I-1, J} P_{I-1, J}^{\prime}+a_{I, J+1} P_{I, J+1}^{\prime}+a_{I, J-1} P_{I, J-1}^{\prime}+b_{I, J}^{\prime}
$$

where

$$
a_{I, J}=a_{I+1, J}+a_{I-1, J}+a_{I, J+1}+a_{I, J-1}
$$


and the coefficients are

$$
\left.\begin{array}{l}
a_{I+1, J}=(d A)_{i+1, J}, \quad a_{I-1, J}=(d A)_{i, J}, \\
a_{I, J+1}=(d A)_{I, j+1}, \quad a_{I, J-1}=(d A)_{I, j}, \\
d_{i, J}=\frac{A_{i, J}}{a_{i, J}}, \quad d_{I, j}=\frac{A_{I, j}}{a_{I, j}}, \quad b_{I, J}^{\prime}=\left(u^{*} A\right)_{i, J}-\left(u^{*} A\right)_{i+1, J}+\left(v^{*} A\right)_{I, j}-\left(v^{*} A\right)_{I, j+1}
\end{array}\right\}
$$

The equation (20) represents the discretized continuity equation evaluated in terms of pressure correction $P^{\prime}$. There is a continuity imbalance in the source term $b^{\prime}$ in this equation due to utilization of the guessed velocities $u^{*}$ and $v^{*}$.

Energy equation: The discretized energy equation at $(\mathrm{I}, \mathrm{J})$ is given by

$$
a_{I, J} \theta_{I, J}=\sum a_{n b} \theta_{n b}
$$

The coefficients of the upwind differencing scheme are

$$
a_{I, J}=a_{I+1, J}+a_{I-1, J}+a_{I, J+1}+a_{I, J-1}+\Delta F
$$

where

$$
\left.\begin{array}{l}
a_{I+1, J}=D_{i+1, J}+\max \left(-F_{i+1, J}, 0\right), a_{I-1, J}=D_{i, J}+\max \left(F_{i, J}, 0\right), \\
a_{I, J+1}=D_{I, j+1}+\max \left(-F_{I, j+1}, 0\right), a_{I, J-1}=D_{I, j}+\max \left(F_{I, j}, 0\right), \\
\Delta F=\left(F_{e}-F_{w}\right)+\left(F_{n}-F_{S}\right)=\left(F_{i+1, J}-F_{i, J}\right)+\left(F_{I, j+1}-F_{I, j}\right)
\end{array}\right\}
$$

and the values of $F$ and $D$ for each of the faces $e, w, n$ and $s$ are defined in a similar manner as given above.

Mass transfer equation: The discretized mass transfer equation at $(I, J)$ is given by

$$
a_{I, J} C_{I, J}=\sum a_{n b} C_{n b}
$$

The coefficients of the upwind differencing scheme are

$$
a_{I, J}=a_{I+1, J}+a_{I-1, J}+a_{I, J+1}+a_{I, J-1}+\Delta F
$$

where

$$
\left.\begin{array}{l}
a_{I+1, J}=D_{i+1, J}+\max \left(-F_{i+1, J}, 0\right), \quad a_{I-1, J}=D_{i, J}+\max \left(F_{i, J}, 0\right), \\
a_{I, J+1}=D_{I, j+1}+\max \left(-F_{I, j+1}, 0\right), a_{I, J-1}=D_{I, j}+\max \left(F_{I, j}, 0\right), \\
\Delta F=\left(F_{e}-F_{w}\right)+\left(F_{n}-F_{S}\right)=\left(F_{i+1, J}-F_{i, J}\right)+\left(F_{I, j+1}-F_{I, j}\right)
\end{array}\right\}
$$

and the values of $F$ and $D$ for each of the faces $e, w, n$ and $s$ are defined in a similar manner as above.

\subsection{Numerical computations}

In order to evaluate the numerical solutions of flow variables $u, v, P, \theta$ and $C$ with relevant parameters $\operatorname{Re}, \operatorname{Pr}, R i$ and $N$, we adopt the SIMPLE algorithm [1] that has been executed by developing a code that runs on a $\mathrm{C}$-compiler. The main steps of this algorithm are given as follows: 


\section{The SIMPLE Algorithm}

The SIMPLE algorithm $[1,21]$ give a method of calculating pressure, velocities, temperature and concentration in a iterative manner, which consists of the following steps:

Step 1: Start with guessed velocities $\left(u^{*}, v^{*}\right)$ pressure fields $P^{*}$, temperature $\theta^{*}$ and concentration $C^{*}$.

Step 2: Calculate the coefficients in the momentum equation, solve discretized momentum equations.

Step 3: Calculate the coefficients of pressure equation, solve pressure correction equations.

Step 4: Correct pressure and velocities:

$$
\left.\begin{array}{l}
P_{I, J}=P_{I, J}^{*}+P_{I, J}^{\prime}, \\
u_{i, J}=u_{i, J}^{*}+d_{i, J}\left(P_{I-1, J}^{\prime}-P_{I, J}^{\prime}\right), \\
v_{I, J}=v_{I, J}^{*}+d_{I, j}\left(P_{I, J-1}^{\prime}-P_{I, J}^{\prime}\right) .
\end{array}\right\}
$$

Step 5: Solve temperature and concentration discretized equations.

Step 6: Replace the previous intermediate values of pressure, velocity, temperature and concentration $\left(P^{*}, u^{*}, v^{*}, \theta^{*}, C^{*}\right)$ with the corrected values $(P, u, v, \theta, C)$, return to Step 2 and repeat this process until the solution converges.

\section{Discussion on results}

In this work, we have investigated steady, free convective flow in a rectangular region with heat and concentration sources using the upwind finite volume scheme. We have chosen the values of different parameters involved in the governing equations such as the Reynolds number, the Prandtl number, the Schmidt number, the Richardson number and the buoyancy ratio and thereby computed $u$-velocity, $v$-velocity, pressure, temperature, concentration and average Nusselt and Sherwood number. The numerical solutions of these flow variables are evaluated by using this scheme with the SIMPLE algorithm. This algorithm helped us to write a code that was executed on the C-compiler and thereby, we have obtained the numerical solutions of the flow variables mentioned above.

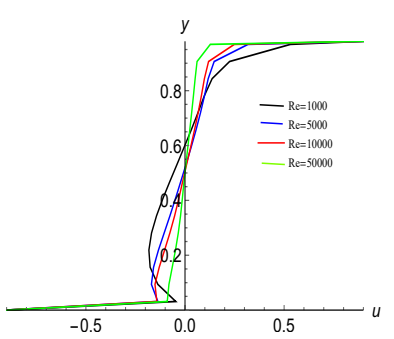

(a)

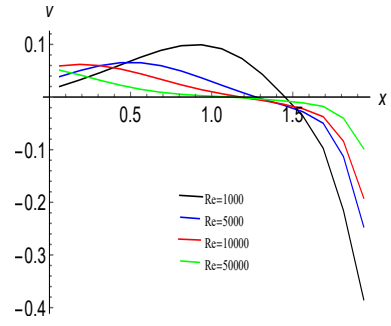

(b)

Fig. 3. (a) $u$-velocity and (b) $v$-velocity profiles for different Reynolds numbers

The numerical solutions of velocity in $\mathrm{x}$-direction (u-velocity) along the vertical line through the geometric centre $(1,0.5)$ of the rectangular domain at different Reynolds numbers 1000, 5000, 10000, 50000, are computed and sketched in Figure 3a. It is observed from this figure that, at $R e=1000$, near the bottom wall, the u-velocity is found to be uniform. 
However, while moving in the upward direction of the geometric centre until the point $y=0.2$, it increases. From there onwards until the point $y=0.6$, the $u$-velocity decreases gradually. Furthermore, from the point $y=0.6$ onwards until the top of the wall, the $u$-velocity is found to be uniformly increasing. So, the intensity of $u$-velocity is observed to be more in the region near the top of the wall. At the top of the wall, it is observed that the $u$-velocity curves for each of the high Reynolds numbers such as 1000,5000,10000,50000 converges and is found to be uniform. The basis for this behaviour of the velocity is that a speed of $u_{0}$ in the positive $x$-direction is given on the top wall. Furthermore, the intensity of the u-velocity at the bottom wall is uniform. However, this behaviour is due to the fact that a heat source whose temperature $T_{h}$ is placed on the bottom wall.

Similarly, by using the numerical solutions obtained for $v$-velocity at different Reynolds numbers $1000,5000,10000,50000$, we have illustrated these values as velocity in the $y$-direction ( $v$-velocity) of the fluid along the horizontal line through the geometric centre $(1,0.5)$ of the rectangular domain in Figure $3 \mathrm{~b}$. It is observed from this Figure that at $R e=1000$, while moving in the horizontal direction from the left wall until the geometric centre, the $v$-velocity increases and reaches its maximum value at the geometric center and, from there onwards, it decreases gradually by cutting the line $y=0.5$ and from there onwards, the $v$-velocity decreases steadily by attaining the lowest value near the right wall. Similarly, for each of the Reynolds numbers 5000,10000,50000, while moving in the horizontal direction of the geometric centre, the $v$-velocity is found to be decreasing. Therefore, the absolute value of the velocity is found to be decreasing with an increase of the Reynolds numbers in the horizontal direction from the left wall until the right wall.

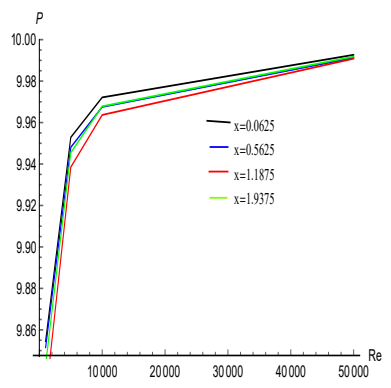

(a)

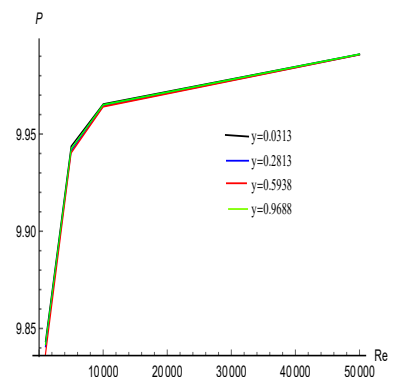

(b)

Fig. 4. Variation of pressure in horizontal and vertical directions for different Reynolds numbers

With the help of the computed numerical solutions of pressure, we have illustrated the variation of fluid pressure at different nodes along the horizontal and vertical directions through the geometric center of the rectangular domain for different Reynolds high numbers in Figure 4. We have sketched the variation of pressure at different Reynolds numbers $1000,5000,10000,50000$ in the range of 1000 to 50000. From the Figure 4a, it is observed that the fluid pressure increases with an increase of the Reynolds numbers for different Reynolds numbers in the range of 1000 to 50000 at four different nodes, as mentioned above, along the horizontal direction through the geometric centre. Similarly, from Figure $4 \mathrm{~b}$ it is observed that the fluid pressure increases with the increase of the Reynolds numbers for different Reynolds numbers in the range of 1000 to 50000 at four different nodes as mentioned above along the vertical direction through the geometric center. The cause for this variation of the pressure in both the directions is due to a heat source being placed on the bottom 
wall. Furthermore, in an enclosed region filled with an incompressible fluid as considered in the present the investigation, when the temperature increases, the pressure of the fluid also increases. This occurs because a heat source is placed on the bottom wall due to which the molecules on the fluid gain more energy and thereby exert more pressure to the walls of the enclosure. Hence, the pressure increases in both directions.



(a) $R e=1000$

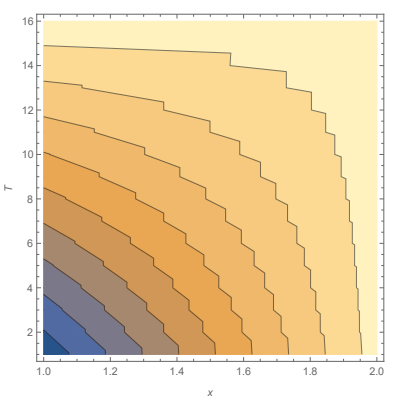

(b) $R e=10000$



(c) $R e=50000$

Fig. 5. Temperature contours along horizontal direction at different Reynolds numbers

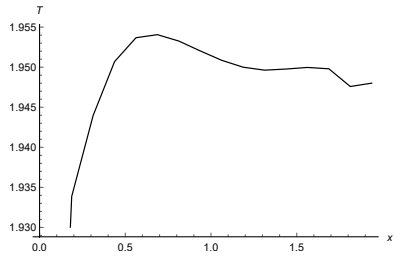

(a) $\operatorname{Re}=1000$

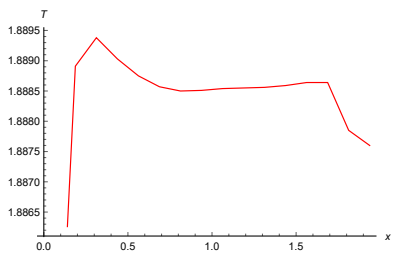

(b) $R e=10000$



(c) $R e=50000$

Fig. 6. Temperature curves along $\mathrm{x}$-axis at different Reynolds numbers

We have chosen the value of the Prandtl number for water as 13.6 to computed the numerical solutions of the temperature variable. With the help of these numerical solutions, we have depicted the nature of the temperature contours and the curves at different Reynolds numbers in the range of 1000 to 50000 along the horizontal direction of the given rectangular domain in Figure 5. From this Figure, it is observed that the flow pattern of the temperature is oscillatory in nature. This nature of the flow pattern is especially more near the right wall rather than at the geometric center of the domain, where it is observed to be less oscillatory. Furthermore, the temperature curves along horizontal direction at different Reynolds numbers are sketched in Figures 6. From these Figures, it has been observed that at three different Reynolds numbers such as $R e=1000,10000,50000$, it is observed that the flow pattern of the temperature is oscillatory in nature. The reason for this has just been explained. Therefore, for high Reynolds numbers as mentioned above, the flow pattern of the temperature is found more oscillatory at the right wall and near the center it was found to be less oscillatory.

We have computed the numerical solutions of the concentration variable by choosing the Schmidt number at 540 that corresponds to incompressible fluids. With the help of these numerical solutions, we have illustrated and elucidated the nature of the concentration contours and curves at different Reynolds numbers in the range of 1000 to 50000 along the horizontal direction of the given rectangular domain in Figure 7. From this Figure, it is observed that the flow pattern of the concentration is oscillatory in nature. This nature of the flow pattern is especially more near the right wall rather than at the geometric center of the domain, where 




(a) $R e=1000$



(b) $R e=10000$

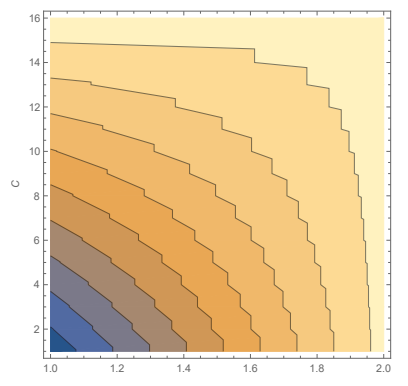

(c) $R e=50000$

Fig. 7. Concentration contours at different Reynolds numbers

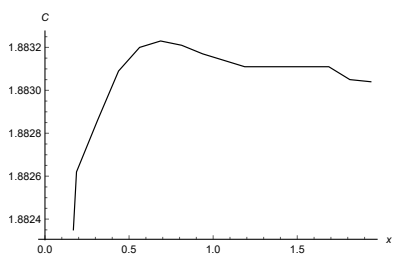

(a) $R e=1000$

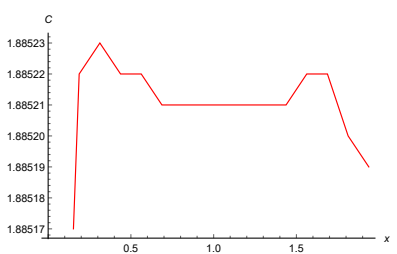

(b) $R e=10000$



(c) $R e=50000$

Fig. 8. Concentration curves along $\mathrm{x}$-axis at different Reynolds numbers

it is observed to be less oscillatory. Furthermore, the concentration curves along horizontal direction at different Reynolds numbers are sketched in Figures 8. From these Figures, it has been observed that at three different Reynolds numbers such as $R e=1000,10000,50000$, it is observed that the flow pattern of the concentration is oscillatory in nature. The reason for this has just been explained. Therefore, for the high Reynolds numbers as mentioned above, the flow pattern of the concentration is found to be more oscillatory at the right wall and near the center it was found to be less oscillatory.

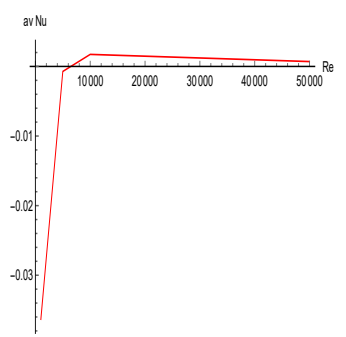

(a)

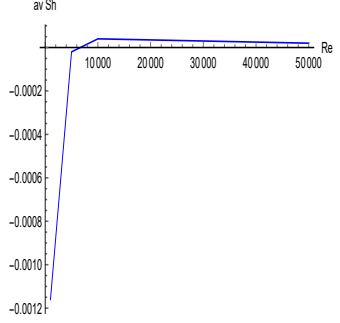

(b)

Fig. 9. Variation of average (a) Nusselt and (b) Sherwood number with the Reynolds numbers

For the purpose of studying the heat and mass transfer from the bottom wall of the rectangular domain, we have computed the numerical solutions of average Nusselt and Sherwood numbers [22]. These values are utilized in sketching the variation of average Nusselt and Sherwood numbers and are given in figure 9. This figure illustrates the average Nusselt number variation versus different Reynolds numbers in the range of 1000 to 50000 . We have observed from this figure that after $R e=1000$, the average Nusselt and Sherwood numbers 
increase and attain their maximum values at $R e=10000$. After that, the average Nusselt and Sherwood numbers remain constant until $R e=50000$. Therefore, the heat and mass transfer at the bottom wall first increases slightly while moving towards the geometric centre in a vertical direction and, after very short distance in a vertical direction, it remains constant and there are no further variations in heat and mass transfers.

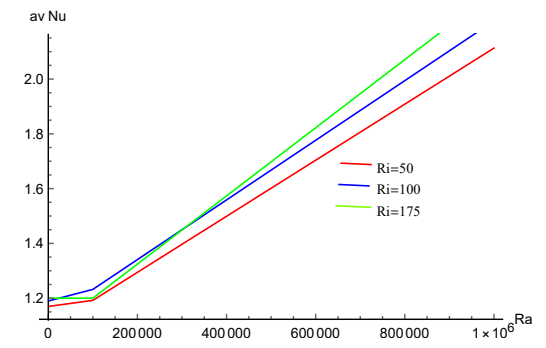

(a)

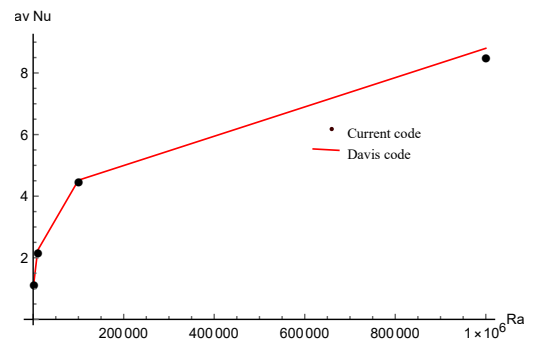

(b)

Fig. 10. (a) Variation of average Nusselt number vs Rayleigh number for different Richardson number and (b) Validation of average Nusselt number with benchmark (Davis) result

We have shown the variation of the average Nusselt number versus Rayleigh number by taking the different Richardson number in Figure 10a. Here, we observed that with an increase in the Richardson number, the average Nusselt number increases with an increase in Rayleigh number. We have validated the values of the average Nusselt number with the benchmark solution in the literature investigated by Davis [3] in Figure 10b. From these comparisons, the solutions obtained from this current investigation are in good agreement with the benchmark solutions available in the literature.

\section{Code validation}

To validate our present computed numerical solutions with the available benchmark solutions in the literature investigated by Ghia et al. [2], we have compared the numerical solutions obtained from the present study for $u$ and $v$ velocities for Reynolds numbers in the range of 1 to 1000 with the solutions of Ghia et al. [2]. From the these comparisons, it is evident that the solutions obtained from the present study are in good agreement with the benchmark solutions existing in the literature.



(a)



(b)

Fig. 11. Validation of the numerical solutions 


\section{Conclusions}

Main conclusions of this study are summarized as follows:

- The intensity of $u$-velocity in the region near the top of the wall was found to be more and also the $u$-velocity curves for each of the high Reynolds numbers such as $1000,5000,10000,50000$ converges there.

- The absolute value of the velocity is found to decrease with the increase of the Reynolds numbers in the horizontal direction from the left wall until the right wall.

- The fluid pressure increases with the increase of the Reynolds numbers for different Reynolds numbers in the range of 1000 to 50000 at four different nodes as mentioned above along the horizontal and vertical directions through the geometric centre.

- For high Reynolds numbers such as $1000,5000,10000,50000$, the flow pattern of the temperature is found to be more oscillatory at the right wall, and near the center it was found to be less oscillatory in both horizontal and vertical directions of the geometric center.

- For high Reynolds numbers such as $1000,5000,10000,50000$, the flow pattern of the concentration is found to be more oscillatory at the right wall and near the center it was found to be less oscillatory in both horizontal and vertical directions of the geometric center.

- It has been found that the heat and mass transfers at the bottom wall first increase slightly while moving towards the geometric centre in a vertical direction and after a very short distance in a vertical direction, it remains constant and there are no further variations in both heat and mass transfers.

- We have validated the values of the average Nusselt number with the benchmark solution in the literature investigated by Davis [3].

\section{Acknowledgments}

The second author acknowledges the financial support received from the University Grant Commission, New Delhi under the scheme of the National Fellowship for Higher Education (NFHE) vide letter no. F1-17.1/2016-17/NFST-2015-17-ST-ASS-2041, dated April, 2016 to carry out this work.

\section{References}

[1] Patankar, S.V., \& Spalding, D.B. (1972). A calculation procedure for heat, mass and momentum transfer in three-dimensional parabolic flows. Int. J. Heat Mass. Transfer, 15(10), 1787-1806.

[2] Ghia, U., Ghia, K.N., \& Shin, C.T. (1982). High-Re solutions for incompressible flow using the Navier-Stokes equations and a multigrid method. Journal of Computational Physics, 48, 387-411.

[3] De Vahl Davis, G. (1983). Natural convection of air in a square cavity: A bench mark numerical solution. International Journal for Numerical Methods in Fluids, 3(3), 249-264.

[4] Bejan, A. (1985). Mass and heat transfer by natural convection in a vertical cavity. Journal of Heat and Fluid Flow, 6(3), 149-159. 
[5] Trevisan, O.V., \& Bejan, A. (1987). Combined heat and mass transfer by natural convection in a vertical enclosure. Journal of Heat Transfer, 109, 104-122.

[6] Phanikumar, M.S. (1994). Thermosolutal convection in a rectangular enclosure with strong sidewalls and bottom heating. International Journal of Heat and Fluid Flow, 15(4), 325-336.

[7] Chamkha, Ali J., \& Al-Naser, H. (2001). Double-diffusive convection in an inclined porous enclosure with opposing temperature and concentration gradients. Int. J. Therm. Sci., 40, 227-244.

[8] Chamkha, Ali J., \& Al-Naser, H. (2002). Hydromagnetic double-diffusive convection in a rectangular enclosure with uniform side heat and mass fluxes and opposing temperature and concentration gradients. International Journal of Thermal Sciences, 41, 936-948.

[9] Ben-Nakhi, A., \& Chamkha, Ali J. (2006). Natural convection in inclined partitioned enclosures. Journal of Heat and Mass Transfer, 42(4), 311-321.

[10] Ben-Nakhi, A., \& Chamkha, Ali J. (2007). Conjugate natural convection in a square enclosure with inclined thin fin of arbitrary length. International Journal of Thermal Sciences, 46, 467-478.

[11] Ben-Nakhi, A., \& Chamkha, Ali J. (2008). Effect of length and inclination of a thin fin on natural convection in a square enclosure. Numerical Heat Transfer, Part A: Applications, 50(4), 381-399.

[12] Teamah, M.A., \& Maghlany, M.E. (2010). Numerical simulation of double-diffusive mixed convective flow in rectangular enclosure with insulated moving lid. International Journal of Thermal Sciences, 49, 1625-1638.

[13] Sathyamoorthy, M., \& Chamkha, Ali J. (2010). Effect of magnetic field on natural convection flow in a square cavity for linearly heated side wall(s). International Journal of Thermal Sciences, 49(9), 1856-1865.

[14] Kuznetsov, G.V., \& Sheremed, M.A. (2011). A numerical simulation of double-diffusive conjugate natural convection in an enclosure. International Journal of Thermal Sciences, 50, $1878-1886$

[15] Ozotop, H.F., Nada, E.A., Varol, Y., \& Chamkha, Ali J. (2011). Natural convection in wavy enclosures with volumetric heat sources. Int. J. Therm. Sci., 50(4), 502-514.

[16] Nikbakhti, R., \& Rahimi, A.B., (2012). Double-diffusive natural convection in a rectangular cavity with partially thermally active side walls. Journal of the Taiwan Institute of Chemical Engineers, 43, 535-541.

[17] Qin, Q., Xia, Z.A., \& Tian, Z.F. (2014). High accuracy numerical investigation of doublediffusive convection in rectangular enclosure with horizontal temperature and concentration gradients. International Journal of Heat and Mass Transfer, 71, 405-423.

[18] Ismael, M.A., Pop, Ioan, \& Chamkha, Ali J. (2014). Mixed convection in a lid-driven square cavity with partial slip. International Journal of Thermal Sciences, 82, 47-61.

[19] Arbin, N., Saleh, H., Hashim, I., Chamkha, Ali J. (2016). Numerical investigation of doublediffusive convection in an open cavity with partially heated wall via heatline approach. International Journal of Thermal Sciences, 100, 169-184.

[20] Ghiaasiaan, Mostafa S. (2011). Convective Heat and Mass Transfer. New York: Cambridge University Press.

[21] Versteeg, H.K., \& Malalasekera, W. (2010). An Introduction to Computational Fluid Dynamics: The Finite Volume Method. Pearson Edition, India.

[22] Bergman, T.L., Lavine, A.S., Incropera, F.P., \& Dewitt, D.P. (2011). Fundamentals of Heat and Mass Transfer, Seventh Edition, John Wiley and Sons, USA. 